\title{
Two new calanoids (Copepoda: Aetideidae) from the abyss of the World Ocean
}

\author{
Ава новых вида каланоид (Copepoda: Aetideidae) \\ из абиссали Мирового океана
}

\author{
Elena L. Markhaseva ${ }^{1}$, Jasmin Renz ${ }^{2}$

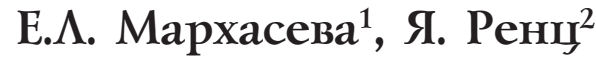

\footnotetext{
${ }^{1}$ Zoological Institute, Russian Academy of Sciences, Universitetskaya nab. 1, St. Petersburg 199034, Russia. E-mail: markhaseva@yahoo.com 2 German Center for Marine Biodiversity Research (DZMB), Senckenberg am Meer, Martin-Luther-King Platz 3 , 20146 Hamburg, Germany

1 Зоологический институт РАН, Университетская наб. 1, Санкт-Петербург 199034, Россия
}

KEY WORDS: Calanoida, Clausocalanoidea, Paracomantenna, Prolutamator, benthopelagic, Kurile-Kamchatka Trench, abyss.

КЛЮЧЕВЫЕ СЛОВА: Calanoida, Paracomantenna, Prolutamator, бентопелагический, Курило-Камчатский желоб, абиссаль.

ABSTRACT. Two new aetideid species, Paracomantenna profunda sp.n. and Prolutamator pseudohadalis sp.n. are described after females collected in the abyss in the vicinity of the sea bed of the World Ocean. Both new species are described from the same type locality in the Kurile-Kamchatka Trench. The new species of Paracomantenna Campaner, 1978 was the most numerous among aetideids in the samples from of the Kurile-Kamchatka Trench and its several specimens were found in the samples from the Weddell Sea and the Atlantic Ocean. Two groups of species are recognized in Paracomantenna and the new species is attributed to the group of the small sized species. It differs from these small sized congeners in the only slightly enlarged surface spines at the maxilla endites and some details of the oral parts setation. Paracomantenna profunda sp.n. shows high variability in the shape of spermathecae and prosome posterior corners and slight variability is observed in the segmentation, setation and ornamentation of the antenna, maxillule, maxilliped and P1. The taxonomic status of the only described male of Paracomantenna is discussed. The genus Prolutamator Markhaseva et Schulz, 2008 is for the first time found in the Pacific Ocean and the new species of the genus from the Kurile-Kamchatka Trench differs from the congeners from the Weddell Sea and the Atlantic Ocean in the larger size, the shape of the spermathecae, and in a very short proximal posterior seta on the maxillule arthrite.

How to cite this article: Markhaseva E.L., Renz J. 2019. Two new calanoids (Copepoda: Aetideidae) from the abyss of the World Ocean // Arthropoda Selecta. Vol.28. No.2. P.213-224. doi: 10.15298/arthsel. 28.2 .04
РЕЗЮМЕ. Два новых вида аэтидеид, Paracomantenna profunda sp.n. и Prolutamator pseudohadalis sp.n. описаны по самкам, собранным в непосредственной близости от дна в абиссали Мирового океана. Оба новых вида описаны из одного типового местонахождения из района Курило-Камчатского желоба. Новый вид рода Paracomantenna Campaner, 1978 был наиболее многочисленным представителем аэтидеид в пробах из Курило-Камчатского желоба и несколько его экземпляров были найдены в пробах из моря Уэддэлла и Атлантического океана. Две группы видов выделяются в роде Paracomantenna и новый вид отнесен группе видов малого размера. От других видов этой группы новый вид отличается мало увеличенными шипами на поверхности эндитов максиллы и некоторыми деталями вооружения ротовых частей. Paracomantenna profunda sp.n. демонстрирует высокую вариабельность формы сперматек и задних углов просомы и небольшую вариабельность в сегментации, вооружении и орнаментации антенны, максиллулы, максиллипеды и Р1. Обсуждается таксономический статус единственного описанного для Paracomantenna самца. Род Prolutamator Markhaseva et Schulz, 2008 в первый раз найден в Тихом океане, а его новый вид из Курило-Камчатского желоба отличается от сородичей из моря Уэддэлла и Атлантического океана большими размерами, формой сперматек и очень короткой проксимальной задней щетинкой артрита максиллулы.

\section{Introduction}

The family Aetideidae represents one of the most taxonomically rich calanoid families and contains over 
200 species in 30 genera [Markhaseva, 1996]. Members of this family are widespread in the World Ocean and inhabit pelagic and benthopelagic environments from shallow to deep waters.

Up to now nine pelagic genera of the family Aetideidae were known from the Kurile-Kamchatka Trench area [Markhaseva, 1996; Markhaseva, Razzhivin, 1992]. The discovery of two more dwelling in the vicinity of the sea bed near-bottom aetideid genera Paracomantenna and Prolutamator in the KuramBio expedition (2012) reveal that aetideid fauna of the Kurile-Kamchatka Trench area is more diverse than considered before.

The genus Paracomantenna Campaner, 1978 contains five species: Paracomantenna goi Ohtsuka, Boxshall et Shimomura, 2005, P. gracilis Alvarez, 1986, P. minor (Farran, 1905), P. magalyae Campaner, 1978, P. wishnerae Markhaseva, 1995 and herein described is $P$. profunda sp.n. Genus Paracomantenna appears to be taxonomically heterogeneous, species split into two groups. First group contain the species $P$. goi and $P$. wishnerae so far recorded from the North Pacific [Markhaseva, 1995; Ohtsuka et al., 2005], that are large $(>2 \mathrm{~mm})$ and share primitive oral parts setation and a P5 present. The second group contain smaller congeners $(<2 \mathrm{~mm})$ inhabiting the Atlantic Ocean, namely $P$. gracilis, $P$. minor and $P$. magalyae that share a similar shape of the posterior prosomal corners and, compared to their larger congeners, are more advanced in the reduced setation of oral parts, and the P5 is absent. The new species from the Kurile-Kamchatka Trench share characters of the smaller congeners (Table 1).

Up to now, all described Paracomantenna species were collected from shallow waters, or from upper slope depths. E.g., P. magalyae and P. gracilis were recorded between 100 and $150 \mathrm{~m}$ [Campaner, 1978; Alvarez, 1986] and P. goi was sampled from $290 \mathrm{~m}$ [Ohtsuka et al., 2005]. The widest distributional vertical range $(300-700 \mathrm{~m})$ was registered for $P$. minor [Farran, 1905; Markhaseva, Schnack-Schiel, 2003], the deepest distribution was observed for $P$. wishnerae, found in depths of $768-825 \mathrm{~m}$ in the bathyal zone above the slope of the seamount Volcano 7 [Markhaseva, 1995]. The new species $P$. profunda is the first representative of Paracomantenna found in the abyssal zone (4069 $\mathrm{m}$ to $5507 \mathrm{~m}$ ) and the first species of the genus with a wide distributional range, including findings from the Atlantic Ocean (between $10^{\circ} \mathrm{N}$ and $16^{\circ} \mathrm{S}$ ), the Weddell Sea and the Kurile-Kamchatka Trench.

The genus Prolutamator includes two species: Pr. hadalis Markhaseva et Schulz, 2008 and Pr. minor Markhaseva et Schulz, 2008. Most Prolutamator specimens were found at abyssal depths of the South Atlantic and the Weddell Sea, while Pr. minor was once collected at a depth of $755 \mathrm{~m}$ in the Southern Ocean [Markhaseva, Schulz, 2008]. The discovery of Pr. pseudohadalis sp.n. is the first record of the genus from the Pacific Ocean.

\section{Material and methods}

Specimens of the described benthopelagic aetideid species were collected close to the sea bed in the abyss of the Pacific, Atlantic and Southern Oceans by a closing epibenthic sledge [Brenke, 2005]. Paracomantenna profunda sp.n. (169 female specimens) was more numerous than Prolutamator pseudohadalis sp.n. (13 female specimens) and the most numerous among aetideids sorted from the samples of the KuramBio expedition collected at 9 stations in 2012 in the Kurile-Kamchatka Trench of the Pacific Ocean. Seven females of Paracomantenna profunda sp.n. were obtained from the Angola and Brazil Basins of the Atlantic Ocean (between $03^{\circ} \mathrm{S}$ and $16^{\circ} \mathrm{S}$ ) during expeditions DIVA-I and DIVA-III in 2000 and 2009, respectively. Two females of $P$. profunda sp.n. were found in samples from the North Atlantic (about $10^{\circ} \mathrm{N}$ ) collected during the Vema-Transit campaign in 2014, and one female was sorted from samples from the Weddell Sea, Southern Ocean, obtained by the ANDEEPII expedition in 2002. The material was fixed in either $96 \%$ pure ethanol or $4 \%$ buffered formalin and later stained by adding a solution of chlorazol black $\mathrm{E}$ dissolved in $70 \%$ ethanol $/ 30 \%$ water. Oral parts and swimming legs were dissected in glycerine and figures were prepared using a camera lucida.

The following abbreviations are used in the descriptions: P1-P5, swimming legs 1-5, Exp 1, exopod segment 1, Enp 1, endopod segment 1. Articulating segments of the antennules are designated by Arabic numerals, ancestral segments by Roman numerals. One seta and 1 aesthetasc on a segment of the antennule are designated: $1 \mathrm{~s}+1$ ae. The number of antennule segments follows Huys \& Bosxhall [1991]. The antenna exopod setation formula is given according to Markhaseva \& Ferrari [2006] and Markhaseva et al. [2014]. Maxilla segments are labeled considering Ferrari \& Ivanenko [2008] as: praecoxal endite (previously considered as proximal praecoxal endite); coxal endite (previously considered as distal praecoxal endite); basal endites (previously considered as coxal endites); enditic-like lobe of proximal endopodal segment (previously considered as basal endite), and the syncoxa of the maxilliped is considered to have 3 praecoxal endites and 1 coxal endite after Ferrari \& Markhaseva [2000 a,b] and Ferrari \& Ivanenko [2001].

Type material and additional specimens are deposited in the Zoological Museum Hamburg (ZMH) and the Zoological Institute, Russian Academy of Sciences, St. Petersburg (ZIN).

\section{Taxonomy}

Family Aetideidae Giesbrecht, 1893

Genus Paracomantenna Campaner, 1978

\section{Paracomantenna profunda sp.n.} Figs 1-4.

HOLOTYPE. Adult female, dissected, body length $1.60 \mathrm{~mm}$. ZMH 45139. Collected above the sea bed in the Kurile-Kamchatka Trench at Sta. $2-10$ on 3 August 2012 by the KuramBio expedition, Sonne cruise SO 223, depths between 4859 and $4865 \mathrm{~m}$.

PARATYPES. 4 adult females, partly dissected, body length $1.50,1.55,1.60$ and $1.70 \mathrm{~mm}$. ZMH 45140, station data as for the holotype; 1.65 and $1.70 \mathrm{~mm}$. ZIN 91133, 2 adult females, station data as for the holotype.

TYPE LOCALITY. $46^{\circ} 14.77^{\prime} \mathrm{N} 155^{\circ} 32.79^{\prime} \mathrm{E}$. 


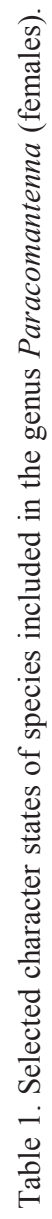

\begin{tabular}{|c|c|c|c|c|c|c|c|c|c|c|c|c|}
\hline 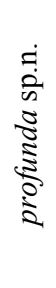 & 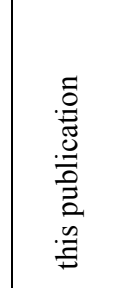 & 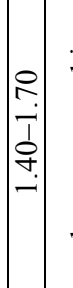 & 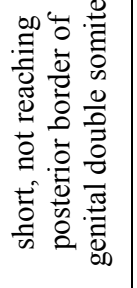 & 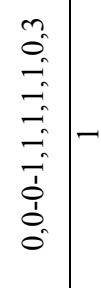 & $\stackrel{n}{\Xi}$ & 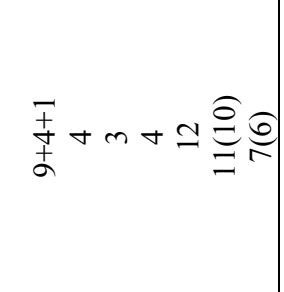 & 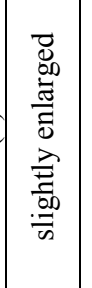 & 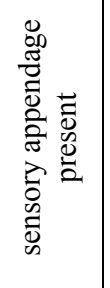 & $\frac{\frac{60}{6}}{6}$ & $\stackrel{8}{9}$ & $\stackrel{8}{2}$ & 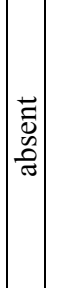 \\
\hline 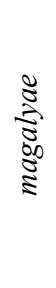 & 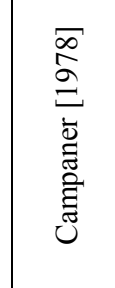 & 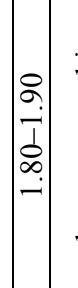 & 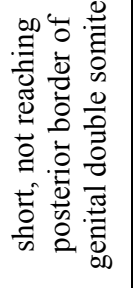 & $\begin{array}{l}0 \\
0 \\
-1 \\
-1 \\
-1 \\
0 \\
0 \\
0 \\
0\end{array}$ & $\frac{9}{2}$ & 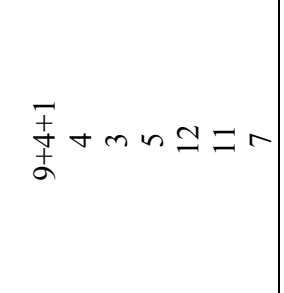 & 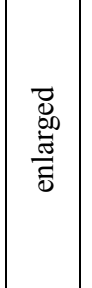 & 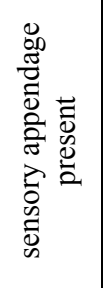 & $\frac{\frac{100}{6}}{6}$ & $\stackrel{8}{g}$ & $\stackrel{\mathscr{D}}{2}$ & 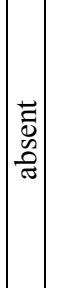 \\
\hline 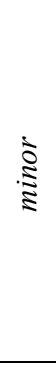 & 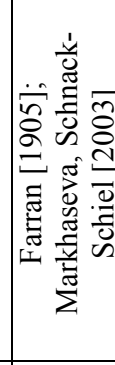 & 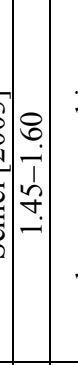 & 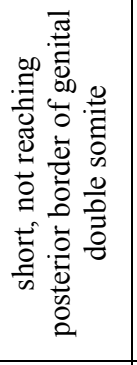 & $\begin{array}{l}0 \\
0 \\
0 \\
= \\
= \\
0 \\
0 \\
0 \\
0 \\
0\end{array}$ & $\stackrel{ \pm}{\Xi}$ & 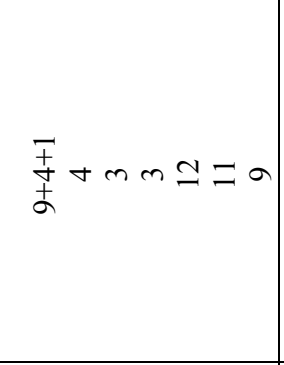 & 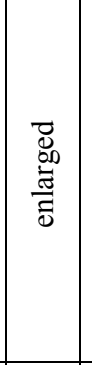 & 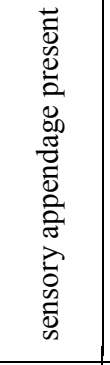 & $\frac{d 0}{0}$ & @ & $\stackrel{D}{2}$ & 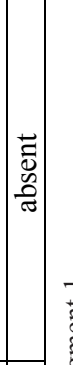 \\
\hline 氶 & 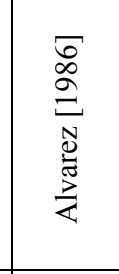 & 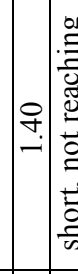 & 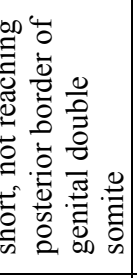 & $\begin{array}{l}3 \\
0 \\
= \\
= \\
0 \\
0 \\
0 \\
0 \\
0\end{array}$ & $\stackrel{n}{\Xi}$ & $\stackrel{f}{S}+m+\simeq=\infty$ & 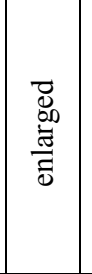 & 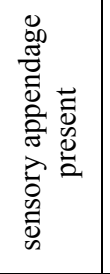 & $\frac{\frac{00}{6}}{6}$ & $\stackrel{\mathscr{D}}{2}$ & $\stackrel{\mathscr{D}}{2}$ & $\mid$ \\
\hline$\tilde{D}_{0}$ & 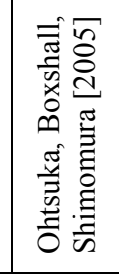 & 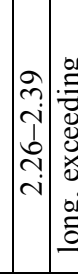 & 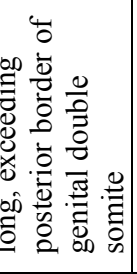 & 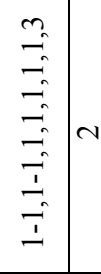 & 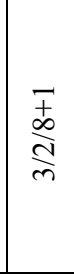 & 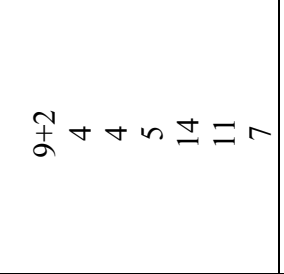 & 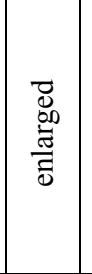 & 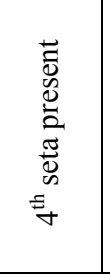 & $\begin{array}{l}\frac{5}{0} \\
\frac{0}{n} \\
\sim \\
\frac{1}{00} \\
\frac{0}{0} \\
0\end{array}$ & 1 & $\stackrel{\circ}{9}$ & 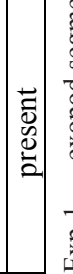 \\
\hline 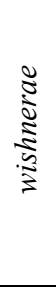 & 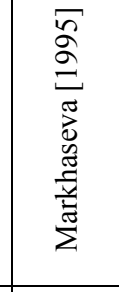 & 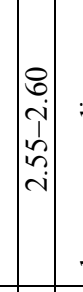 & 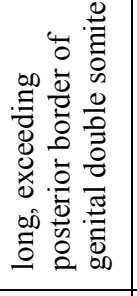 & 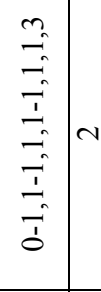 & $\frac{m}{3}$ & 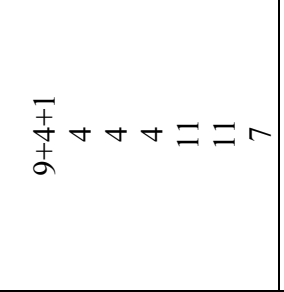 & 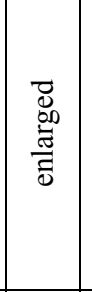 & 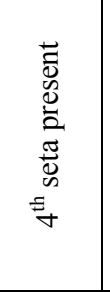 & 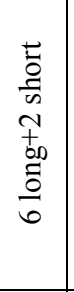 & 1 & @ & 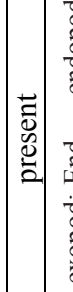 \\
\hline 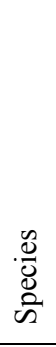 & 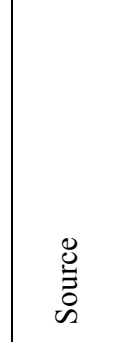 & 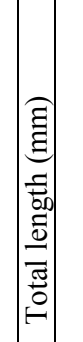 & 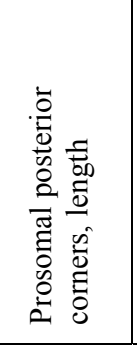 & 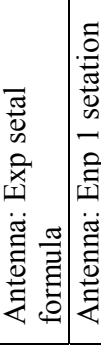 & 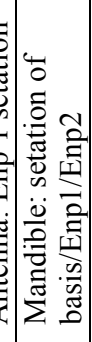 & 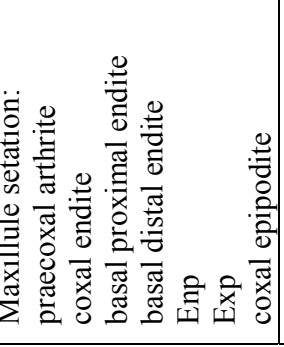 & 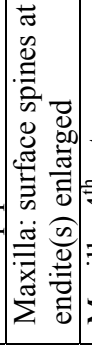 & 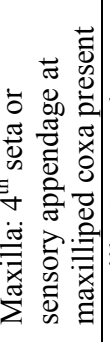 & & 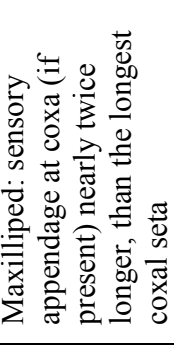 & 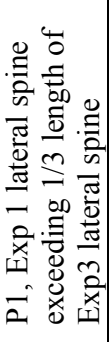 & 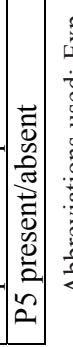 \\
\hline
\end{tabular}




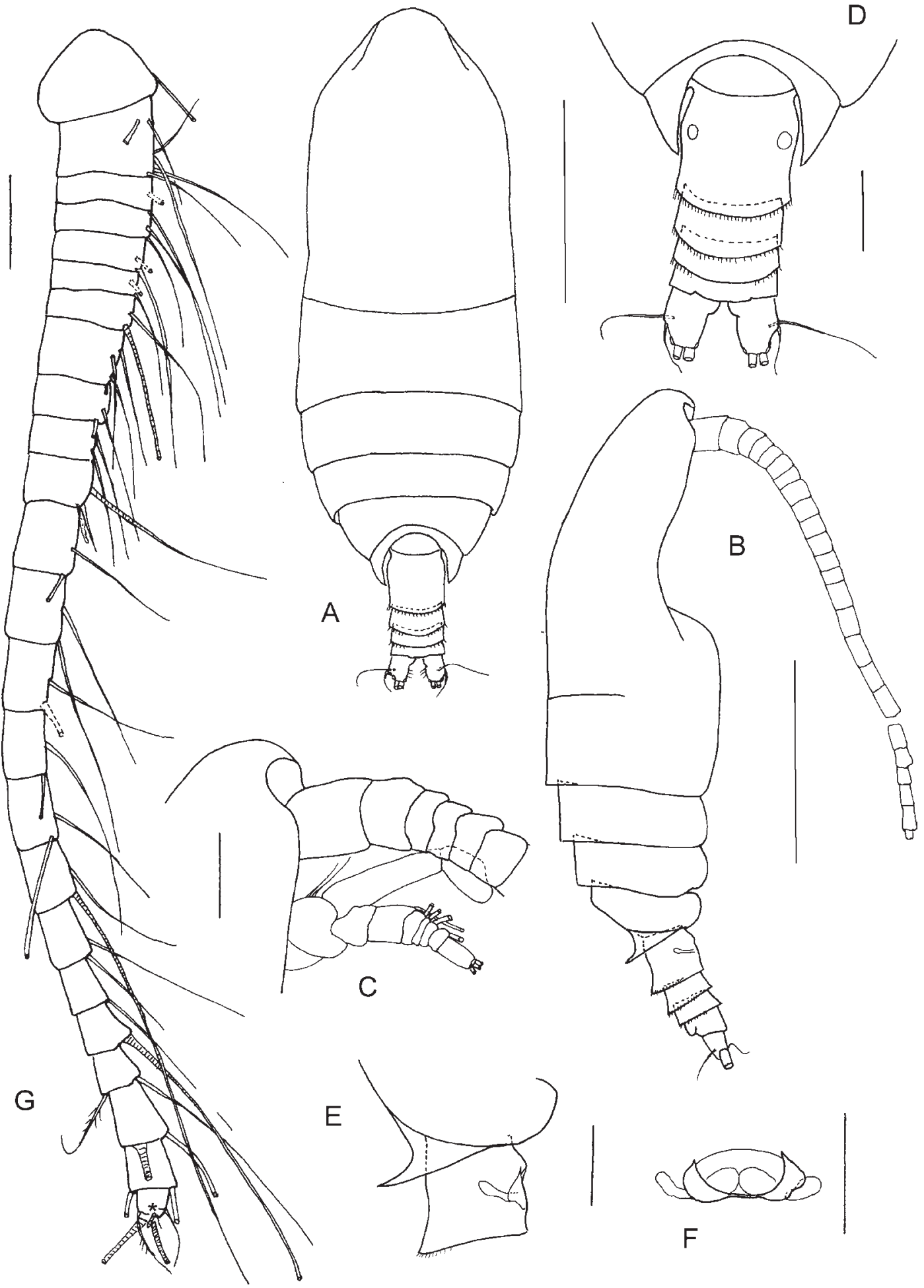

Fig. 1. Paracomantenna profunda sp.n. Female, holotype. A - habitus, dorsal view; B - habitus, lateral view; C - cephalosome, lateral view; D - posterior prosome and urosome, dorsal view; E - posterior prosome and genital double somite, lateral view; F — genital field, ventral view; $\mathrm{G}$ - antennule, dotted line marks additions after another holotype limb; star marks aesthetasc.

Scale bars: A-B $-0.5 \mathrm{~mm}, \mathrm{C}-\mathrm{G}-0.1 \mathrm{~mm}$.

Рис. 1. Paracomantenna profunda sp.n. Самка, голотип. А - общий вид дорсально; В - общий вид латерально; С цефалосома латерально; D - задняя часть просомы и уросома дорсально; Е - задняя часть просомы и генитальный сомит латерально; F — гениальное поле вентрально; G - антеннула, пунктиром показаны дополнения по другой антеннулы голотипа; звездочкой отмечен эстетаск.

Масштаб: А-B - 0,5 мм, $\mathrm{C}-\mathrm{G}-0,1$ мм. 


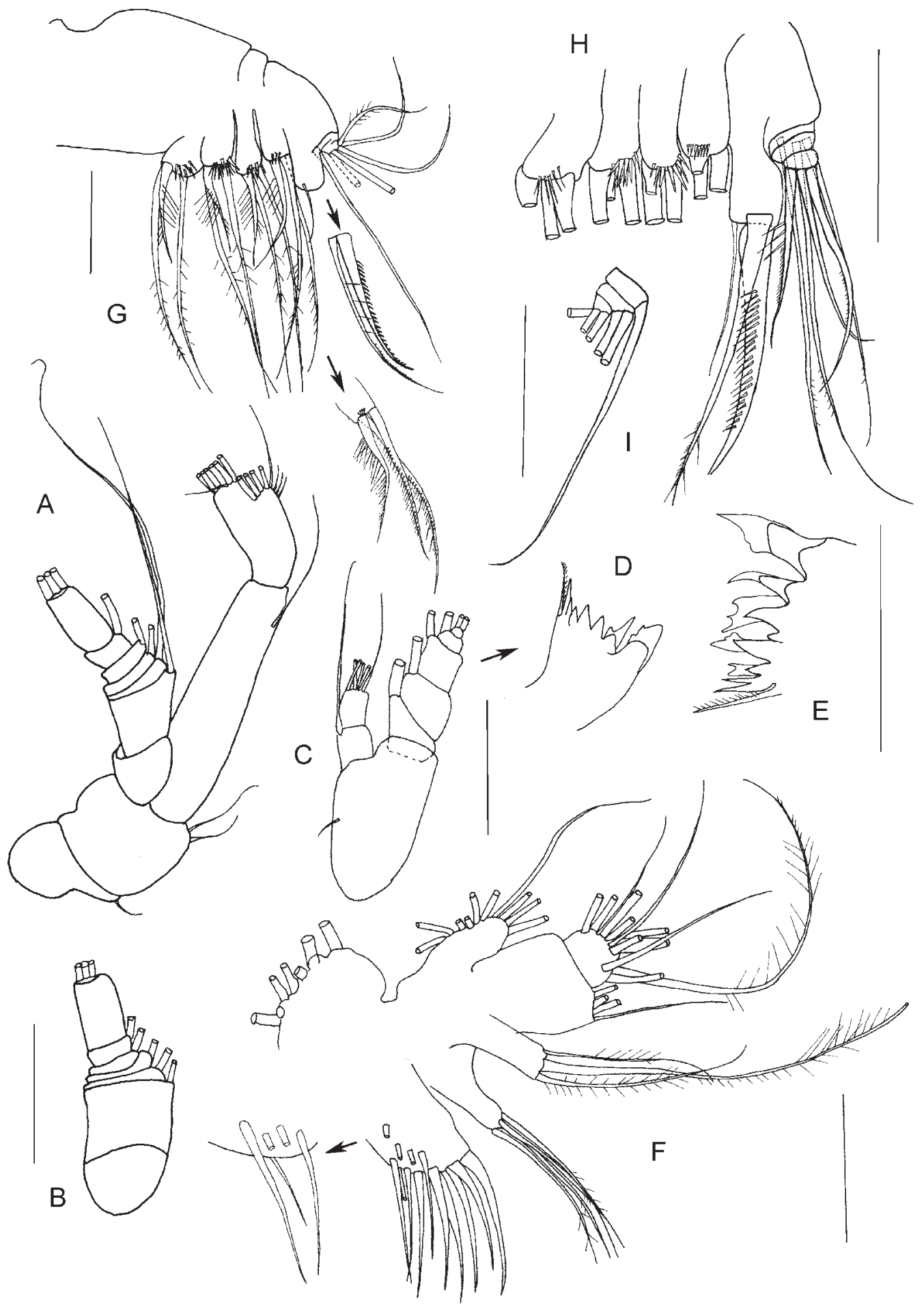

Fig. 2. Paracomantenna profunda sp.n. Female. A — antenna; B - antenna exopod, different position; C - mandible, palp; D, E mandible, gnathobase cutting edge; $\mathrm{F}$ - maxillule, arrow marks praecoxal arthrite, only posterior and anterior setae figured; $\mathrm{G}$ - maxilla, dotted line marks additions after another holotype limb, arrows mark basal endite setae and setae of enditic-like lobe of endopod; $\mathrm{H}$ maxilla endites and endopod; I - maxilla endopod.

A-D, F-G - holotype, H - paratype, E, I - specimen from the Kurile-Kamchatka Trench, Sta. 5-10. Scale bars $0.1 \mathrm{~mm}$.

Рис. 2. Paracomantenna profunda sp.n. Самка. А — антенна; В - экзоподит антенны, другой ракурс; С — пальпа мандибулы; $\mathrm{D}, \mathrm{E}$ - режущий край гнатобазы мандибулы; F — максиллула, стрелка указывает на деталь артрита на которой показаны только задние и передняя щетинки; G - максилла, пунктиром показаны добавления по второй конечности голотипа, стрелки указывают на щетинки базального эндита и щетинки лопасти эндоподита; Н - максилла, эндиты и эндоподит; I — максилла, эндоподит.

A-D, F-G - голотип, H - паратип, E, I - экземпляры из района Курило-Камчатского желоба, Ст. 5-10. Масштаб 0,1 мм. 


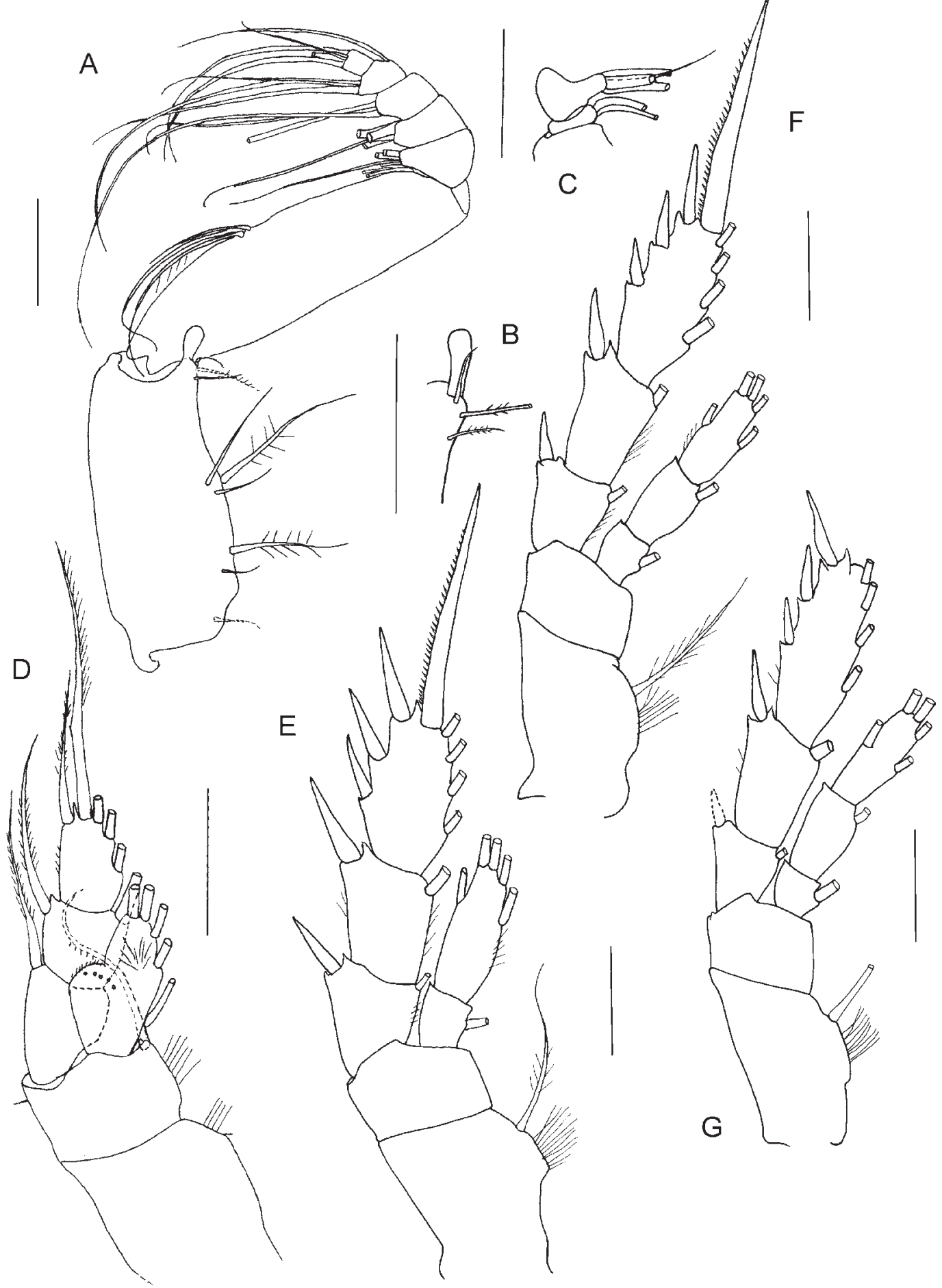

Fig. 3. Paracomantenna profunda sp.n. Female. A - maxilliped, dotted line marks additions after another holotype limb; B maxilliped, coxal setae and sensory appendage; $\mathrm{C}$ - maxilliped, endopod segments 1 and $2 ; \mathrm{D}-\mathrm{P} 1$, dotted line marks additions after another holotype limb; E - P2; F, P3; G - P4, left, dotted line marks additions after right paratype P4.

A, D - holotype, B - specimen from the Kurile-Kamchatka Trench, KuramBio expedition; Sta. 7-10, C - specimen from Northern Atlantic, Vema-Transit expedition, Sta. 2-7; E-G - paratype. Scale bars $0.1 \mathrm{~mm}$.

Pис. 3. Paracomantenna profunda sp.n. Самка. А - максиллипеда, пунктиром показаны дополнения по другой максиллипеде голотипа; В - максиллипеда, щетинки коксы и сенсорный придаток; С - максиллипеда, сегменты эндоподита 1 и 2 ; D - P1, пунктиром показаны дополнения по другой ноге голотипа; $\mathrm{E}-\mathrm{P} 2 ; \mathrm{F}-\mathrm{P} 3 ; \mathrm{G}-\mathrm{P} 4$, левая, пунктиром показаны дополнения по правой ноге паратипа.

A, D - голотип, В — экземпляр из Курило-Камчатского желоба, экспедиция KuramBio, Ст. 7-10, С — экземпляр из Северной Атлантики, экспедиция Vema-Transit, Ст. 2-7; E-G - паратип. Масштаб 0,1 мм. 

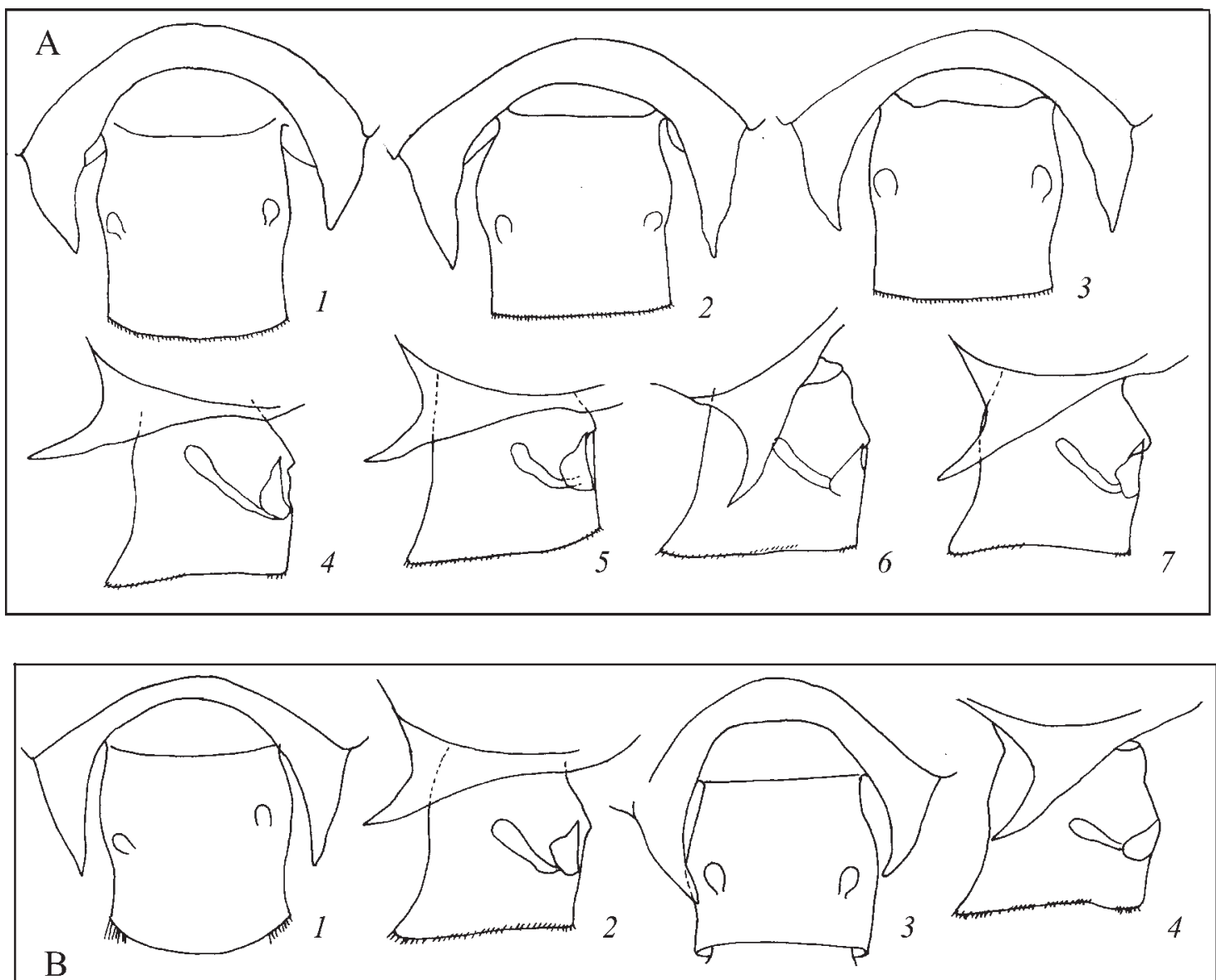

$\mathrm{B}$
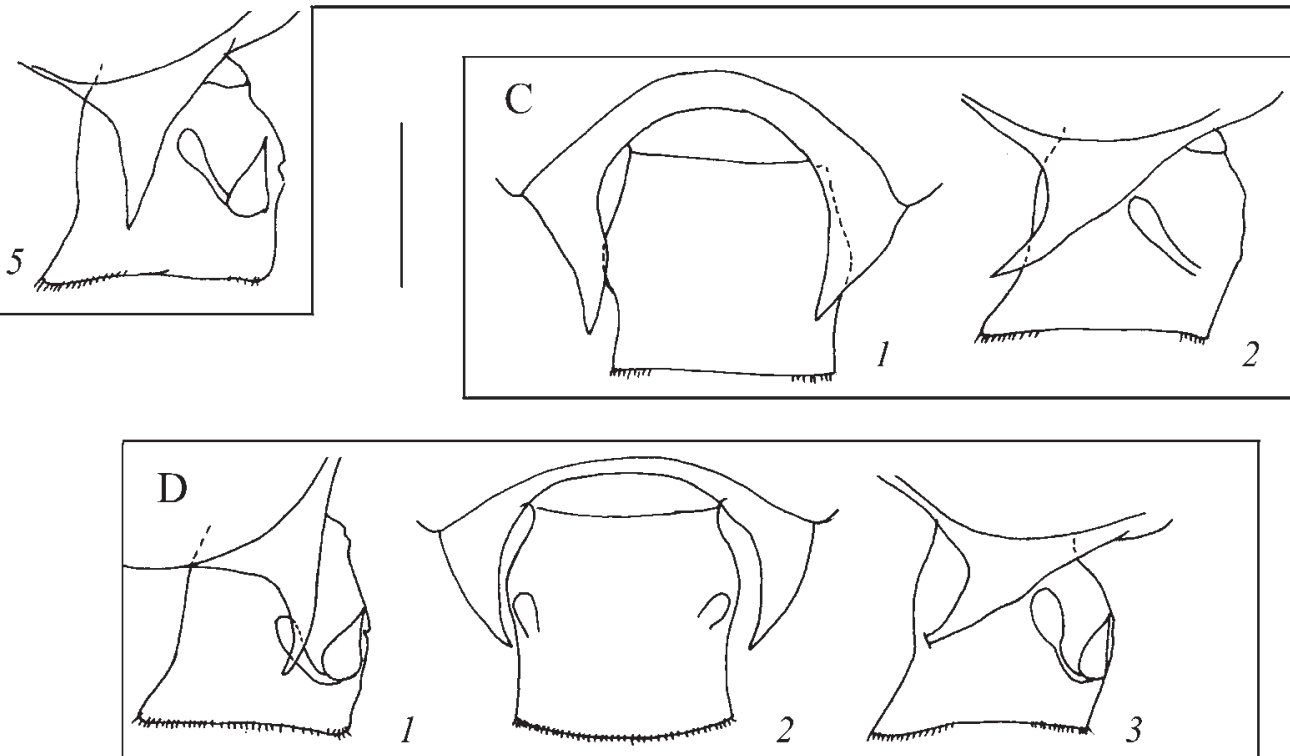

Fig. 4. Paracomantenna profunda sp.n. Posterior prosome and double genital somite in dorsal and lateral views. A — specimens from the Pacific Ocean, Kurile-Kamchatka Trench, KuramBio expedition: 1 and 3-7 - Sta. 7-10 and 2-Sta. 5-10; B - specimens from the Atlantic Ocean: 1-2 - Brazil Basin, DIVA-III expedition, Sta. 609 and 3-5 - Angola Basin, DIVA-I expedition, Sta. 348; C - specimen from the Southern Ocean, Weddell Sea, ANDEEP-II expedition, Sta. 134; D — specimens from the Atlantic Ocean, North Atlantic, VemaTransit expedition, Sta. 2-7.

Рис. 4. Paracomantenna profunda sp.n. Задняя часть просомы и генитальный сомит дорсально и латерально. А — экземпляры из Тихого океана, Курило-Камчатский желоб, экспедиция KuramBio: 1 и 3-7 - Ст. 7-10 и $2-$ Ст. 5-10; В - экземпляры из Атлантического океана: 1-2 - Бразильский бассейн, экспедиция DIVA-III, Ст. 609 и 3-5 - Ангольский бассейн, экспедиция DIVA-I, Ст. 348; C - экземпляры из Южного океана, море Уэддэлла, экспедиция ANDEEP-II, Ст. 134; D — экземпляры из Атлантического океана, северная Атлантика, экспедиция Vema-Transit, Ст. 2-7. 
ADDITIONAL MATERIAL. 162 females, collected in the Kurile-Kamchatka Trench, Pacific Ocean, KuramBio expedition, Sonne cruise SO 223. Two females, body length $1.45 \mathrm{~mm}$ and 1.40 mm, Sta. $1-11$, 30 July $2012,43^{\circ} 58.44^{\prime} \mathrm{N} 157^{\circ} 18.29^{\prime} \mathrm{E}$, depths $5418-5419 \mathrm{~m}$; 6 females, body length $1.50 \mathrm{~mm}$ (5 females) and $1.55 \mathrm{~mm}$ ( 1 female), Sta. $5-10,11$ August $2012,43^{\circ} 34.44^{\prime} \mathrm{N}$ $153^{\circ} 58.06^{\prime} \mathrm{E}$, depths $5375-5378 \mathrm{~m}$; 6 females, Sta. 6-12, $15 \mathrm{Au}-$ gust $2012,42^{\circ} 28.49^{\prime} \mathrm{N} 153^{\circ} 59.54^{\prime} \mathrm{E}$, depths $5304-5307 \mathrm{~m} ; 73$ females, body length of 33 females $1.45-1.60 \mathrm{~mm}$, Sta. 7-10, 17 August $2012,43^{\circ} 01.82^{\prime} \mathrm{N} 152^{\circ} 58.55^{\prime} \mathrm{E}$, depths $5223-5221 \mathrm{~m} ; 27$ females, Sta. $8-12,21$ August $2012,42^{\circ} 14.38^{\prime} \mathrm{N} 151^{\circ} 43.12^{\prime} \mathrm{E}$, depths 5127-5124 m; 24 females, Sta. 9-12, 24 August 2012, $40^{\circ} 34.49^{\prime} \mathrm{N} 150^{\circ} 59.85^{\prime} \mathrm{E}$, depths $5399-5392 \mathrm{~m} ; 15$ females, Sta. $10-12,27$ August $2012,41^{\circ} 12.80^{\prime} \mathrm{N} 150^{\circ} 05.61^{\prime} \mathrm{E}$, depths 5245 $5262 \mathrm{~m} ; 9$ females, Sta. $11-12,31$ August $2012,40^{\circ} 12.32^{\prime} \mathrm{N}$ $148^{\circ} 05.73^{\prime} \mathrm{E}$, depths $5350-5348 \mathrm{~m}$.

Nine females were collected above the sea bed from the Atlantic Ocean. Six females, body length 1.5 and $1.60 \mathrm{~mm}$ from the Angola Basin, DIVA-I expedition, RV Meteor cruise ME 48-1, Sta. 348,27 July $2000,16^{\circ} 16.98^{\prime} \mathrm{S} 5^{\circ} 27.27^{\prime} \mathrm{W}$, depth $5390 \mathrm{~m}$. One female was collected from the Brazil Basin, DIVA-III expedition, RV Meteor cruise ME 79-1, Sta. 609, 6 August 2009, 357.54'S $5^{\circ} 028.03 .07^{\prime} \mathrm{W}$, depth $5170 \mathrm{~m}$. Two females, body length $1.50 \mathrm{~mm}$, were collected in the North Atlantic, Vema-Transit SO 237, Sta. 2$7,20.12 .2014,10^{\circ} 42.89^{\prime} \mathrm{N} 25^{\circ} 03.21^{\prime} \mathrm{W}$, depth $5507 \mathrm{~m}$.

One female, body length $1.65 \mathrm{~mm}$, was collected above the sea bed from the Southern Ocean, Weddell Sea, ANDEEP-II expedition, RV Polarstern cruise ANT XIX, Sta. 134, 9 March 2002, $65^{\circ} 19.20^{\prime} \mathrm{S} 48^{\circ} 03.81^{\prime} \mathrm{W}$, depth $4069 \mathrm{~m}$.

DESCRIPTION. Female. Body length $1.40-1.70 \mathrm{~mm}$. Prosome 3.3-4.2 times as long as urosome (Fig. 1A-B). Rostrum absent (Fig. 1B, C). Cephalosome and pedigerous somite 1 incompletely separate, and pedigerous somites 4 and 5 completely separate; posterior corners in dorsal view triangular; in lateral view pointed, directed dorsally (Fig. 1B, E, 4 A-D). Urosome of 4 somites (Fig. 1A-B, D). Spermathecae upturned anteriorly, shape variying (Fig. 1E$\mathrm{F}, 4 \mathrm{~A}-\mathrm{D})$. Caudal rami with 1 dorsal seta, 1 ventral seta, and 4 terminal setae.

Antennule (Fig. 1G) of 24 articulating segments, reaching pedigerous somite 3 , or exceeding to somites 4-5; armature as follows: I-1s, II-IV-6s, V to IX-2s each, X-XI-4s, XII and XIII-2s each, XIV-2s + 1ae, XV to XXI-2s each, XXII and XXIII-1s each, XXIV-XXVI-2s each, XXVIIXXVIII-5s + 1ae.

Antenna (Fig. 2A-B), coxa with 1 seta, basis with 2 setae; exopod of 8 segments with $0,0-0-1,1,1,1,1,0$, and 3 setae, all terminal setae long, of nearly the same length; first endopodal segment with 1 seta, second with $8+7$ setae.

Mandible (Fig. 2C-E), gnathobase with 4 large and 3 small teeth near dorsal seta; basis with 1 seta; exopod 5segmented with $1,1,1,1$, and 2 setae; endopod segment 1 with 1 seta, segment 2 with 5 setae.

Maxillule (Fig. 2F), praecoxal arthrite with 9 terminal, 4 posterior and 1 anterior setae, coxal endite with 4 setae; coxal epipodite with 6-7 setae; proximal basal endite with 3 setae, distal basal endite with 4 setae; endopod with 12 setae; exopod with 10-11 setae.

Maxilla (Fig. 2G-I), praecoxal endite with 3 setae; coxal endite with 3 setae; basal endites with 3 setae each, all endites decorated with denticles that are most developed at coxal and proximal basal endites; enditic-like lobe of proximal endopodal segment with 3 setae, one seta thicker, spinelike; endopod with $6(1+2+3)$ setae.

Maxilliped (Fig. 3A-C), syncoxa with 1 seta on proximal praecoxal endite, 2 setae on middle praecoxal endite and 3 setae on distal praecoxal endite; coxal lobe with 3 setae and sensory appendage. Basis with 3 setae. Endopod 6- segmented with $2,4,4,3,3+1$, and 4 setae, first segment very small.

Swimming legs. P1 (Fig. 3D), coxa with medial spinules; basis with medial spinules, small lateral spinule distally and medial distal seta curved with setules; endopod 1-segmented with lateral lobe, its lateral margin usually with spinules and pores, anterior segment surface with distal spinules; exopod segments 1 to 3 with 1 long lateral spine each, spines of exopod segments 1 and 2 reaching nearly to the distal third if the following spine. P2-P4 (Fig. 3E-G), coxa with 1 seta and medial spinules; basis without seta; endopod 2-segmented in P2, 3-segmented in P3-P4; exopods 3-segmented. P5 absent.

ETYMOLOGY. The species name "profunda" refers to the depth where species was discovered. Gender feminine.

REMARKS. The new species is closely related to the species of the Paracomantenna small sized species group, viz. $P$. gracilis, $P$. minor and $P$. magalyae (Table 1). $P$. profunda sp.n. shares the mandible palp setal formula with $P$. gracilis, but differs from this species in the maxillule coxal epipodite setation as 6 or 7 setae ( $v s 8$ setae in $P$. gracilis) and in the smaller size of the sensory appendage at the maxilliped coxa (shorter than the longest coxal seta $v s$ twice longer then the longest coxal seta in $P$. gracilis). The new species differs from $P$. minor and $P$. magalyae in the 5 setae that are present at the mandible endopod segment 2 ( $v s$ 4 setae present in $P$. minor and P. magalyae); the maxillule distal basal endite that is supplied by 4 setae ( $v s 3$ or 5 setae in P. minor and P. magalyae respectively), and in some other details of the oral part setation (Table 1).

Specimens of the new species show slight variability in the setation of the maxillule, P1 endopod ornamentation and in the segmentation of the antenna exopod and maxilliped endopod. E.g., both maxillule usually have exopods supplied by 11 setae and coxal epipodites supplied by 7 setae. However, in some specimens, $P$. profunda sp.n. possesses a maxillule with 10 setae at one of exopods (holotype and the specimen from the Brazil Basin), or 6 setae at one of the coxopod epipodites (holotype, one specimen from the KurileKamchatka Trench and the specimen from the Brazil Basin). In the specimen from the Angola Basin, compared to the holotype, the anterior seta of the maxillule praecoxal arthrite is longer. Usually the antenna exopod segments 2 and 3 supplied by the first and second proximal setae are separate, however, these segments were found to be incompletely separate in one specimen from the North Atlantic. Also in the other specimens from the North Atlantic the maxilliped endopod segment 1 was separated from the basis, while it was fused to the basis in the other specimens (Fig. 3A, C). Variability was also expressed in the P1 endopod ornamentation, i.e., in some specimens from the Kurile-Kamchatka Trench pores and hairs at P1 endopod were well observed, but were not visible in the other specimens (Fig. 3D).

Unlike the relatively slight variabilities in the limbs armament and segmentation, strong variations were found in the shape of spermathecae and prosome posterior corners both, between specimens from the Kurile-Kamtchaka Trench (Figs 1E, 4A, 1-7), and between specimens from the other oceans (Fig. 4B, 1-5; C, 1-2; D, 1-3).

\section{Prolutamator pseudohadalis sp.n.} Figs 5-6.

HOLOTYPE. Adult female, dissected, body length $3.40 \mathrm{~mm}$. ZMH 45136. Collected above the sea bed in the Kurile-Kamchatka 

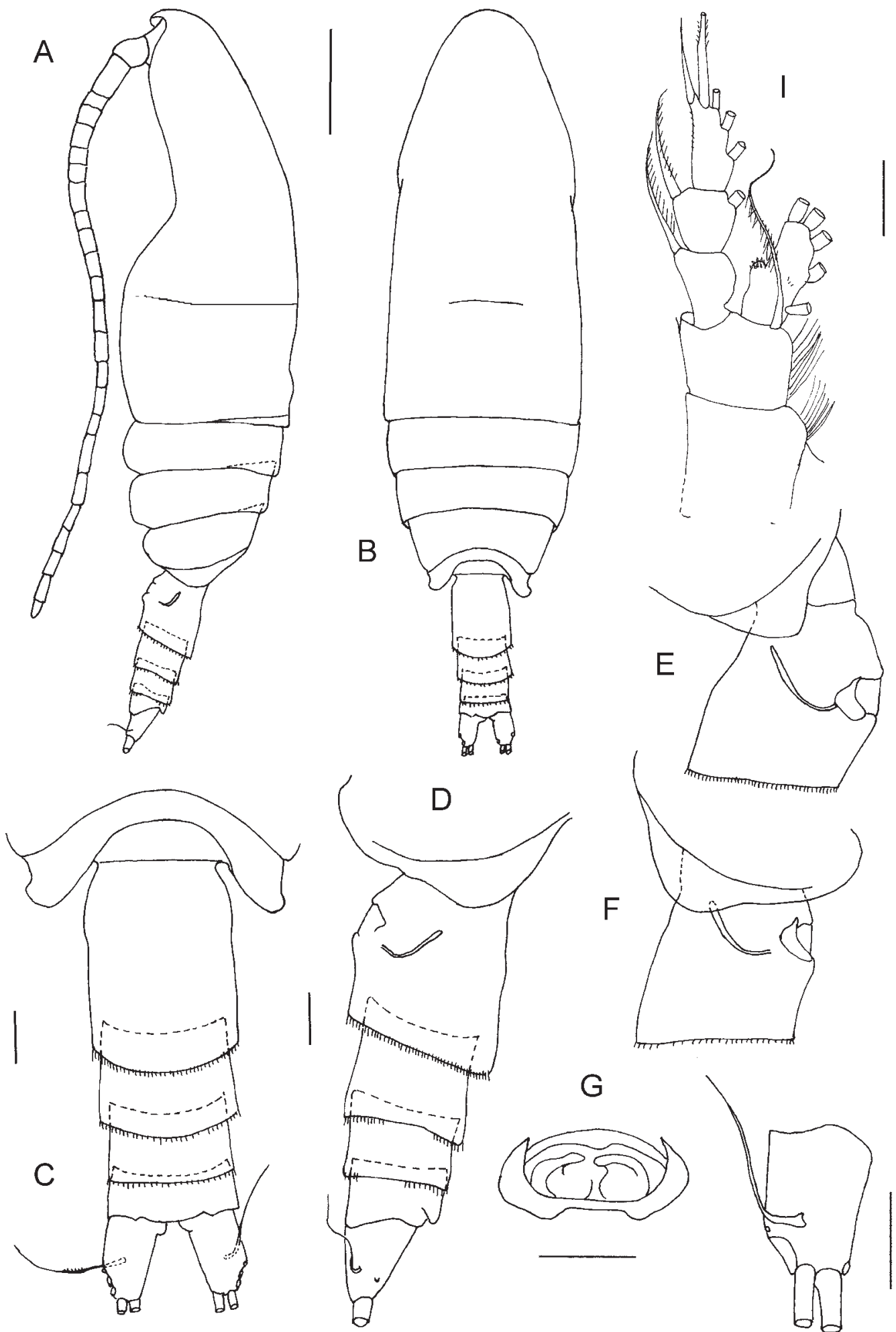

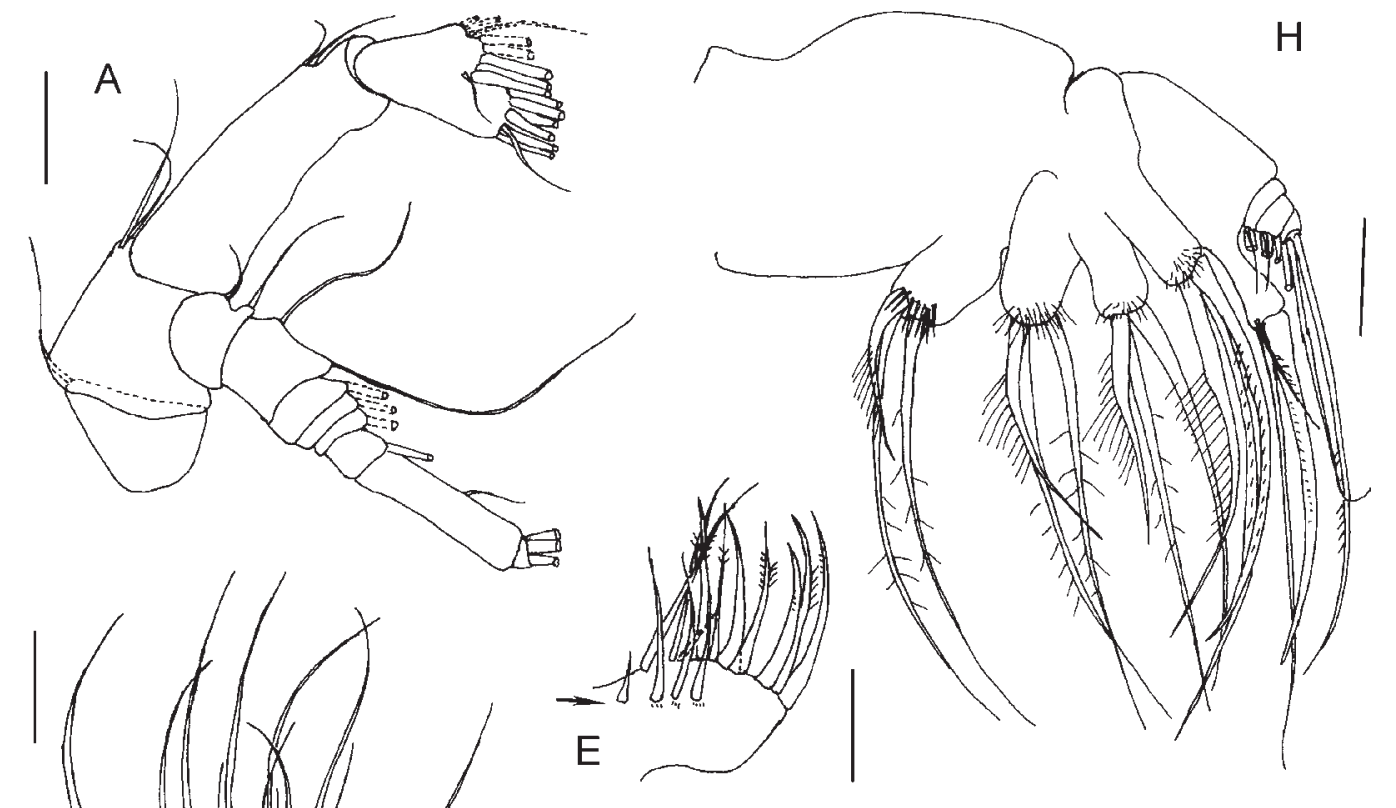

$\mathrm{H}$

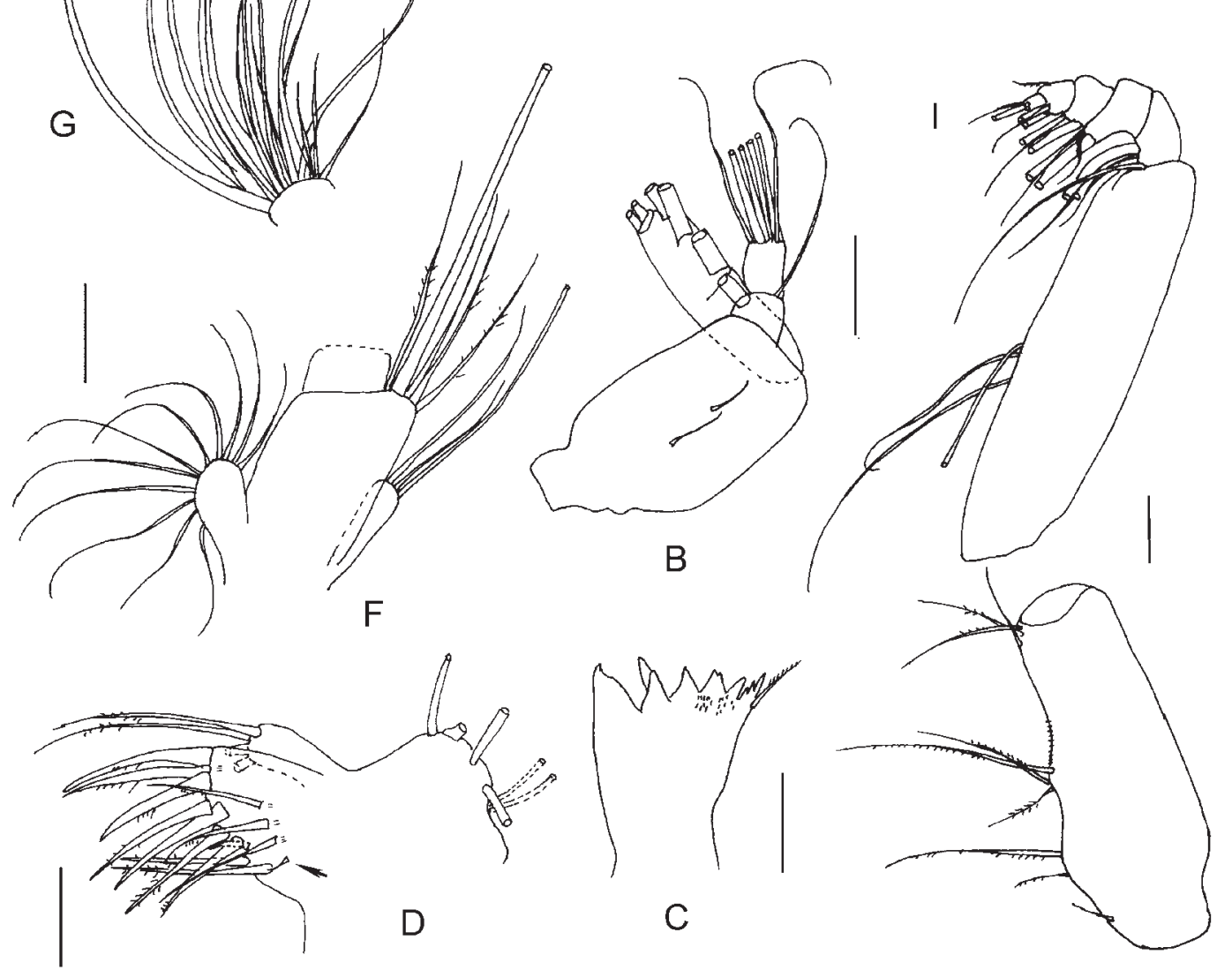

Fig. 6. Prolutamator pseudohadalis sp.n. Female. A — antenna, dotted line marks setae added after paratype; B — mandible, palp; C — mandible, gnathobase cutting edge; D - maxillule, praecoxal arthrite, coxal endite and epipodite, arrow marks small proximal posterior praecoxal arthrite seta; E - maxillule, praecoxal arthrite, arrow marks small proximal posterior seta; F — maxillule, basal endites, endopod and exopod, setae of endopod not figured; G - maxillule, distal part of endopod with setae; H - maxilla; I - maxilliped.

A-D, F-I - holotype, E - paratype. Scale bars $0.1 \mathrm{~mm}$.

Рис. 6. Prolutamator pseudohadalis sp.n. Самка. А - антенна, пунктиром обозначены щетинки добавленные по антенне паратипа; В - мандибула, щупик; C - мандибула, край гнатобазы; D - максиллула, прекоксальный артрит, коксальный эндит и эпиподит, стрелка указывает на маленькую проксимальную заднюю щетинку; Е — максиллула, прекоксальный артрит, стрелка указывает на маленькую проксимальную заднюю щетинку прекоксального артрита; F — максиллула, базальные эндиты, эндоподит и экзоподит, щетинки эндоподита не нарисованы; G — максиллула, дистальная часть эдоподита со щетинками; Н - максилла; I - максиллипеда.

A-D, F-I — голотип, E — паратип. Масштаб 0,1 мм. 
Trench at Sta. $2-10$ on 3 August 2012 by the KuramBio expedition, Sonne cruise SO 223, depths between 4859 and $4865 \mathrm{~m}$.

PARATYPES. 4 adult females, partly dissected, body length $3.40,3.45,3.50$ and $3.55 \mathrm{~mm}$. ZMH 45137, station data as for the holotype; 1 adult female, body length $3.40 \mathrm{~mm}$. ZIN 91132. Collected above the sea bed in the Kurile-Kamchatka Trench at Sta. 510, 11 August 2012, by the KuramBio expedition, Sonne cruise SO 223 , depths $5375-5378 \mathrm{~m}, 43^{\circ} 34.44^{\prime} \mathrm{N} 153^{\circ} 58.06^{\prime} \mathrm{E}$.

TYPE LOCALITY. $46^{\circ} 14.77^{\prime} \mathrm{N} 155^{\circ} 32.79^{\prime} \mathrm{E}$.

ADDITIONAL MATERIAL. 7 females, collected from the Kurile-Kamchatka Trench, Pacific Ocean, KuramBio expedition, Sonne cruise SO 223. 2 females, body length $3.40 ; 3.50 \mathrm{~mm}$; Sta $6-12,15$ August $2012,42^{\circ} 28.49^{\prime} \mathrm{N} 153^{\circ} 59.54^{\prime} \mathrm{E}$, depths 5304 $5307 \mathrm{~m} ; 4$ females, Sta. $7-10,17$ August $2012,43^{\circ} 01.82^{\prime} \mathrm{N}$ $152^{\circ} 58.55^{\prime} \mathrm{E}$, depths $5223-5221 \mathrm{~m}$; 1 female, Sta. 8-12, 21 August $2012,42^{\circ} 14.38^{\prime} \mathrm{N} 151^{\circ} 43.12^{\prime} \mathrm{E}$, depths $5127-5124 \mathrm{~m}$

DESCRIPTION. Female. Body length $3.40-3.55 \mathrm{~mm}$. Prosome 3.2-3.4 times as long as urosome (Fig. 5A-B). Rostrum absent (Fig. 5A). Cephalosome and pedigerous somite 1 incompletely separate, and pedigerous somites 4 and 5 completely separate; posterior corners in dorsal view oval, in lateral view rounded (Fig. 5A-F). Urosome of 4 somites (Fig. 5A-D). Spermathecae upturned, very narrow, in ventral view not visible. (Fig. $5 \mathrm{D}, \mathrm{E}-\mathrm{F}$ ). Caudal rami with 1 dorsal seta, 1 ventral seta, and 4 terminal setae $(5 \mathrm{H})$.

Antennule (Fig. 5A) reaching posterior border of genital double somite, of 24 articulating segments; armature in holotype as follows: I-1s, II-IV-6s, V to VI-2s each, VII$1 \mathrm{~s}+1$ ?, VIII-2s, IX-2s ( 1 seta long), X-XI-4s, XII to XVI$2 \mathrm{~s}$ each, XVII-1s+1?, XVIII- XIX-2s each, XX-2s (1 seta long), XXI-2s+1ae, XXII-1s, XXIII-1s (1 seta long), XXIVXXV-2s each, XXVI-2s (1 seta long) and XXVII-XXVIII $-4 \mathrm{~s}+1 \mathrm{ae}(5 \mathrm{~s}$ in paratype specimen).

Antenna (Fig. 6A), coxa with 1 seta, basis with 2 setae; exopod of 8 articulated segments with 1-1, 1-1, 1, 1, 1, 1, 1, and 3 setae; first endopodal segment with 2 setae, second with $8+7$ setae.

Mandible (Fig. 6B-C), gnathobase with 5 large and 3 small teeth near dorsal seta; basis with 2 setae; exopod 5segmented with $1,1,1,1$, and 2 setae; endopod segment 1 with 1 seta, segment 2 with 6 setae.

Maxillule (Fig. 6D-G), praecoxal arthrite with 9 terminal, 4 posterior and 1 anterior setae, one posterior seta small; coxal endite with 4 setae; coxal epipodite with 6 setae; proximal basal endite with 3 setae, distal basal endite with 5 setae; endopod with 15 setae; exopod with 11 setae.

Maxilla (Fig. 6H), praecoxal endite with 3 setae; coxal endite with 3 setae; basal endites with 3 setae each, all endites decorated with thin denticles; enditic-like lobe of proximal endopodal segment with 3 setae, one seta thicker, spine-like; endopod with 6 long and 2 thin small setae.

Maxilliped (Fig. 6I), syncoxa with 1 seta on proximal praecoxal endite, 2 setae on middle praecoxal endite and 3 setae on distal praecoxal endite; coxal lobe with 3 setae. Basis with 3 setae. Endopod 6-segmented, 2 setae of the first segment fused to basis, and following segments with 4, 4, 3, $3+1$, and 4 setae.

Swimming legs. P1 (Fig. 5I), coxa with medial spinules; basis with medial spinules, small lateral spinule distally and medial distal curved seta with setules; endopod 1-segmented with lateral lobe, its lateral margin with spinules, posterior segment surface with few tiny spinules; exopod segments 1 to 3 with 1 long lateral spine each, spine of exopod segments 1 reaching nearly to the distal third of the following spine, spine of exopod segment 2 reaching the first third of the following spine. P2-P4, coxa with 1 seta and medial spinules; basis without seta; endopod 2-segmented in P2, 3-segment- ed in P3-P4; exopods 3-segmented and as figured for Pr. hadalis [Markhaseva, Schulz, 2008, Fig. 4B-C]. P5 absent.

ETYMOLOGY. The species name "pseudohadalis" refers to the close relation to the species Prolutamator hadalis. Gender masculine.

REMARKS. The new species differs from the other species of Prolutamator in the larger size $(3.40-3.55 \mathrm{~mm} v \mathrm{~s}$ 2.70-2.90 $\mathrm{mm}$ in Pr. hadalis and $2.25-2.35 \mathrm{~mm}$ in $P r$. minor), in the shape of the spermathecae, which is narrow along its entire length ( $v s$ spermathecae is widened with its anterior part oval-rounded in congeners), and in a very short proximal posterior seta at the maxillule arthrite ( $v s$ all maxillule arthrite posterior setae are long in congeners). $P r$. pseudohadalis sp.n. is more closely related to the species Pr. hadalis and shares with Pr. hadalis the setation of the mandible basis with 2 setae and the maxillule epipodite with 6 setae, but differs by a small distal knob that is absent from the prosome posterior lobe ( $v s$ knob is present in Pr. hadalis).

\section{Discussion}

The aetideid genus Prolutamator is not numerous and comprises three morphologically uniform species, whereas it is not that for the genus Paracomantenna. Two groups of species are recognized in the genus Paracomantenna. The first group contains species $>2$ $\mathrm{mm}$ ( $P$. goi and $P$. wishnerae) and the second group unites species $<2 \mathrm{~mm}(P$. gracilis, $P$. minor, $P$. magalyae and the new species $P$. profunda described herein). The smaller species differ from their larger congeners in the following morphological characters: 1) the prosomal posterior corners short, not reaching posterior border of the genital double somite ( $v s$ long, exceeding posterior border of the gential double somite in larger species); 2) the antenna exopod setal formula is $0,0-0-1,1,1,1,1,0,3$ (vs $1-1,1-1,1,1,1,1,1,3$ or $0-1,1$ $1,1,1-1,1,1,3$ in larger species); 3 ) the mandible basis with one seta, or seta absent; endopod segment 1 with one seta, and endopod segment 2 with 4 or 5 setae ( $v s$ respectively: 3, 2 and 7 or $8+1$ setae in larger species); 4) the maxillule proximal basal endite with 3 setae (vs 4 setae in larger species); 5) the maxilla endopod with 6 long setae ( $v s 6$ long and 2 short, small setae in larger species); 6) the maxilliped coxa with 3 setae and the sensory appendage present ( $v s 4$ setae, the sensory appendage lacking in larger species); 7) the P1 exopod segment 1 lateral spine reaching, often exceeding one third length of the exopod segment 3 lateral spine ( $v S$ not reaching one third length of the exopod segment 3 lateral spine in larger species), and 8) the P5 absent (vs P5 present in larger species) (Table 1).

Species of Paracomantenna, that were described up to now are rare, mostly known after few individuals and only infrequently after tens of specimens [Campaner, 1978]. Despite plenty of adults of the new species (169 specimens) found in the Kurile-Kamchatka Trench samples, no males were discovered among them. Up to now, the only adult male attributed to the genus Paracomantenna is that of P. minor [Markhaseva, Schnack-Schiel, 2003]. Attribution of this male to the 
genus Paracomantenna rather than to Bradyidius Giesbrecht, 1897 was questioned by Ohtsuka et al. [2005]. While this male resembles species of Bradyidius, its assignment to this genus is doubtful due to the lacking rostrum, that is usually present and biramous in Bradyidius. Furthermore, this male possesses a very short antenna exopod (about 0.6 of endopod length) which is typical for Paracomantenna, while the exopod of Bradyidius is longer than the endopod. Uncertainty in the attribution of the $P$. minor male to Paracomantenna arises due to the absence of the sensory appendage at its maxilliped coxa, as the sensory appendage is typically present in females of the same species. However, this morphological difference between $P$. minor sexes [Markhaseva, Schnack-Schiel, 2003] may express their sexual dimorphism. Similar differences in the possession of the sensory appendage at the maxilliped coxa can be observed in the females (sensory appendage present) and males (sensory appendage absent) of $\mathrm{Co}$ mantenna brevicornis (Sars 1902), a species of a genus that is closely related to Paracomantenna [Matthews, 1964; Alvarez, 1986; Markhaseva, 1996].

Further revision of Paracomantenna is necessary to resolve interspecific relationships within the genus. The complete revision of the genus, however, is only possible with a future discovery of males of other species, which are currently lacking.

Diagnosis of the morphologically homogeneous genus Prolutamator awaits male description. So far only one highly dimorphic male specimen without biological name was found among numerous females and preliminary attributed to the genus [Renz, Markhaseva 2015].

Rare reports of the aetideid benthopelagic males found in the near-bottom may be due to their high sexual dimorphism that makes difficult their taxonomical assignment or to sex-specific differences in behavior, where males are distributed higher up in the water column to avoid being eaten by other predators [Renz, Markhaseva 2015] and this sex-specific distribution would prohibit sampling of males by epibenthic sledges.

Acknowledgements. The authors thank Prof. Pedro Martínez Arbizu and Prof. Angelika Brandt for providing the calanoid the specimens described in this paper. Elena Markhaseva acknowledges the ZIN theme AAAAA-A17117030310207-3 and her research at the German Center for Marine Biodiversity Research (DZMB), which was funded by a stipendium of the Research Institute Senckenberg. This work benefitted from the sharing of expertise within the DFG priority program SPP 1991 Taxon-Omics and support from DFG RE RE2808/3-1.

\section{References}

Alvarez M.P.J. 1986. New calanoid copepods (Aetideidae) of the genera Comantenna, Mesocomantenna, new genus, and Para- comantenna off the Brazilian coast // Journal of crustacean biology. Vol.6. P.858-877.

Campaner A.F. 1978. On some new planktobenthic Aetideidae and Phaennidae (Copepoda, Calanoida) from the Brazilian continental shelf. I. Aetideidae // Ciência e Cultura. Vol. 30. No.7. P.863-876.

Farran G.P. 1905. Report on the Copepoda of the Atlantic slope off counties Mayo and Galway // Annual Report Fisheries Ireland 1902-1903, (2), App. 2. Scientific Investigations. P.23-52.

Ferrari F.D., Ivanenko V. 2001. Interpreting segment homologies of the maxilliped of cyclopoid copepods by comparing stagespecific changes during development // Organisms, Diversity and Evolution. Vol.1. P.113-131. http://dx.doi.org/10.1078/ 1439-6092-00009

Ferrari F.D., Ivanenko V. 2008. The identity of protopodal segments and the ramus of maxilla 2 of copepods (Copepoda) // Crustaceana. Vol.81. P.823-835. http://dx.doi.org/10.1163/ 156854008784771702

Ferrari F.D., Markhaseva E.L. 2000a. Brachycalanus flemingeri and B. brodskyi, two new copepods (Crustacea: Calanoida: Phaennidae) from benthopelagic waters of the tropical Pacific // Proceedings of the Biological Society of Washington. Vol.113. P.1064-1078.

Ferrari F.D., Markhaseva E.L. 2000b. Grievella shanki, a new genus and species of scolecitrichid calanoid copepod (Crustacea) from a hydrothermal vent along the South East Pacific Rise // Proceedings of the Biological Society of Washington. Vol.113. P.1079-1088.

Huys R., Boxshall G.A. 1991. Copepod Evolution. London: The Ray Society. 468 p. http://dx.doi.org/10.1163/193724092x00193

Markhaseva E.L. 1995. A new species of Paracomantenna from the eastern tropical Pacific (Crustacea, Copepoda: Aetideidae) // Zoosystematica Rossica. Vol.3. No.2. P.259-233.

Markhaseva E.L. 1996. Calanoid copepods of the family Aetideidae of the world ocean // Proceedings of the Zoological Institute RAS, St. Petersburg. Vol.268. 331 p.

Markhaseva E.L., Ferrari F.D. 2006. New benthopelagic bradfordian calanoids (Crustacea: Copepoda) from the Pacific Ocean with comments on generic relationships // Invertebrate Zoology. Vol.2 (for 2005). No.2. P.111-168.

Markhaseva E.L., Laakmann S., Renz J. 2014. An interim synopsis of the Bradfordian families with a description of Thoxancalanus spinatus (Copepoda: Calanoida), a new diaixid genus and species from the deep Atlantic Ocean // Marine Biodiversity. Vol.44. P.63-88. http://dx.doi.org/10.1007/s12526-013-0185-0

Markhaseva E.L., Razzhivin V. 1993. Vertical distribution of copepods of the family Aetideidae (Copepoda, Calanoida) in the vicinity of the Kurile-Kamchatka Trench // Oceanology. Vol.32. No.5. P.611-617.

Markhaseva E.L., Schnack-Schiel B. 2003. New and rare calanoid copepods from the Great Meteor Seamount, North Eastern Atlantic // Ophelia. Vol. 57. No.2. P.107-123.

Matthews J.B.L. 1964. On the biology of some bottom-living copepods (Aetideidae and Phaennidae) from western Norway // Sarsia. Vol.16. P.1-46.

Ohtsuka S., Boxshall G.A., Shimomura M. 2005. Three new species of deep-sea hyperbenthic Aetideid copepods (Crustacea) collected from Nansei Islands, southwestern Japan // Hasegawa K., Shinohara G., Takeda M. (eds.). Deep-Sea Fauna and Pollutants in Nansei Islands. National Science Museum Monographs. Vol.29. P.225-247.

Renz J., Markhaseva E.L. 2015. First insights into genus level diversity and biogeography of deep sea benthopelagic calanoid copepods in the South Atlantic and Southern Ocean // DeepSea Research Part I. Vol. 105. P.96-110.

Responsible editor K.G. Mikhailov 